\title{
Levantamento qualitativo de gêneros de parasitos em amostras fecais de jacarés criados comercialmente em sistema fechado no estado do Rio de Janeiro ${ }^{1}$
}

\begin{abstract}
André M.B. Batista ${ }^{2}$, Maria A.V. Da Costa Pereira ${ }^{2 *}$, Gilmar F. Vita ${ }^{3}$, Celso G. Barbosa ${ }^{4}$, Isabela M. Silva Antonio ${ }^{2}$, Suzana C.W. Barros ${ }^{2}$, Alex R. Magalhães ${ }^{3}$ e Juliana P. Freitas ${ }^{3}$

ABSTRACT.- Batista A.M.B., Da Costa Pereira M.A.V., Vita G.F., Barbosa C.G., Silva Antonio I.M., Barros S.C.W., Magalhães A.R. \& Freitas J.P. 2012. [Qualitative survey of parasites genera on fecal samples of alligators in a commercial closed farming system in the state of Rio de Janeiro.] Levantamento qualitativo de gêneros de parasitos em amostras fecais de jacarés criados comercialmente em sistema fechado no estado do Rio de Janeiro. Pesquisa Veterinária Brasileira 32(10):1045-1049. Setor de Parasitologia, Hospital Veterinário, Laboratório de Sanidade Animal, Universidade Estadual do Norte Fluminense Darcy Ribeiro, Av. Alberto Lamego 2000, Campos dos Goytacazes, RJ 28013-602, Brazil. E-mail: angelicadacostapereira@yahoo.com.br

The objective of this paper was to diagnose qualitatively parasite genera found in environmental fecal samples of alligators (Caiman latirostris Daudin, 1802) commercially bred from 2008 to 2009 in a closed farming system in the state of Rio de Janeiro. A total of 300 samples were collected from 150 young, 80 fattening and 70 breeding processed by two different methods, the flotation (method of Willis-Mollay) and simple sedimentation (method of Lutz), according to Hoffmann (1987). The samples were then examined by optical microscopy. The results revealed presence of Eimeria and Isospora oocysts, Balantidium cysts, and Acanthostomum and Dujardinascaris eggs.
\end{abstract}

INDEX TERMS: Caiman sp., commercial breeding, qualitative diagnose, parasites.

RESUMO.- 0 objetivo desta pesquisa foi realizar um diagnóstico qualitativo dos gêneros de parasitos encontrados em amostras fecais ambientais de jacarés (Caiman latirostris Daudin, 1802), criados comercialmente em sistema fechado, no período de 2008 a 2009, no estado do Rio de Janeiro. Um total de 300 amostras foi coletado de 150 filhotes, 80 de animais de engorda e 70 de reprodução, e submetido a análises coproparasitológicas, de flutuação (método de Willis-Mollay) e sedimentação simples (método de Lutz), de acordo com Hoffmann (1987). As amostras foram

\footnotetext{
${ }^{1}$ Recebido em 20 de dezembro de 2011.

Aceito para publicação em 30 de maio de 2012

${ }^{2}$ Setor de Parasitologia, Hospital Veterinário, Laboratório de Sanidade Animal, Universidade Estadual do Norte Fluminense Darcy Ribeiro (UENF), Av. Alberto Lamego 2000, Campos dos Goytacazes, RJ 28013-602, Brasil. *Autor para correspondência: angelicadacostapereira@yahoo.com.br

${ }^{3}$ Programa de Pós-Graduação em Biologia Animal, Instituto de Biologia, Universidade Federal Rural do Rio de Janeiro (UFRRJ), Seropédica, Universidade Castelo Branco (UCB), Rua José Túnula 8, Seropédica, RJ 23890000, Brasil.

${ }^{4}$ Departamento de Matemática, Universidade Federal Rural do Rio de Janeiro (UFRRJ), Seropédica, RJ 23890-000.
}

visualizadas à luz da microscopia óptica. Os resultados obtidos evidenciaram a presença de oocistos de Eimeria e Isospora, cistos de Balantidium e ovos de Acanthostomum e Dujardinascaris.

TERMOS DE INDEXAÇÃO: Caiman sp., criação comercial, diagnóstico qualitativo, parasitos.

\section{INTRODUÇÃO}

Os jacarés, crocodilos e aligátores surgiram na Terra há pelo menos 200 milhões de anos, compartilhando o planeta com os dinossauros. No Brasil ocorrem somente representantes da Subfamília Alligatorinae, sendo que as espécies de jacarés mais incidentes são o jacaré-açu (Melanosuchus niger Spix, 1825), que é o maior deles e vive na Amazônia, o jacaré-do-paraguai ou jacaretinga (Caiman yacare Daudin, 1802), cujos olhos são circundados por listas e o jacaré-de- papo-amarelo (Caiman latirostris Daudin, 1802), chamado assim por possuir sua região ventral de tonalidade amarela esbranquiçada, encontrado na região que compreende do Rio São Francisco até o sul do país. Todos os jacarés americanos são muito parecidos entre si, sendo que o maior deles 
é o jacaré-paguá (Palesouchus palpebrosus Cuvier, 1807), que vive na América do Sul (Catto 1991, Sarkis-Gonçalves et al. 2001, Mourão \& Campos 2004).

Como quaisquer seres vivos, os répteis são susceptíveis a uma grande variedade de fatores intrínsecos, como por exemplo, temperatura corporal/ambiental, e fatores extrínsecos, infestação ou infecção por parasitos. No caso dos crocodilianos, existe mais de uma centena de espécies de helmintos, principalmente trematódeos e nematódeos. No parasitismo ou parasitose, na maioria das vezes os sinais clínicos são inespecíficos, incluindo perda de peso de forma progressiva, decréscimos nos níveis de atividade e em alguns casos, diarréias e até anorexia (Catto 1991, Huchzermeyer 2003, Jaques 2011).

O conhecimento a respeito da fauna parasitológica dos répteis ainda é um campo de pesquisa a ser explorado. Já existem alguns trabalhos abordando parasitas de répteis, como tartarugas, serpentes, entre outros, uma vez que vem crescendo a demanda destes animais como animais de estimação, se fazendo necessário maior conhecimento a respeito destes, como comportamento, fisiologia, doenças e tratamentos (Panizzutti et al. 2003, Dias et al. 2004, Foreyt 2005).

A caça exploratória de $C$. latirostris, com finalidade de obtenção de carne e couro de excelente qualidade, bem como a destruição do ambiente natural destes animais contribui de forma grave para seu desaparecimento da fauna brasileira. A caça indiscriminada destes animais vem sendo combatida por pessoas conscientes da importância dos mesmos tanto no aspecto cultural, quanto no aspecto ecológico. Esta caça é estimulada pelo alto valor comercial aferido ao couro destes répteis, que é o mais caro existente.

A criação comercial legalizada destes animais vem tendo a finalidade tanto de preservação da espécie, já que havendo locais legalizados para a criação destes, reduz-se muito sua caça, como também, uma forma de garantir o fornecimento de carne e couro para o mercado consumidor cada vez mais exigente, seletivo, e com possibilidade de criação de novas divisas para o país.

Justifica-se o estudo devido à existência de poucos trabalhos na literatura mundial, onde quase todos se restringem a descrições de espécies, sem informações sobre a ocorrência, intensidade de infecção ou interações hospedeiro-parasito-ambiente.

Tendo em vista a grande variedade de atributos ligada a estes animais, cabe a nós atentar para a necessidade da preservação dos mesmos, já que todos os anos milhares destes répteis são vítimas das ações humanas, diretas e indiretas.

Somente através de pesquisas sérias e muito trabalho será possível garantir que estes animais que habitam a Terra há mais de 70 milhões de anos continuem presentes nos rios e lagos, e não como lembranças, carteiras e sapatos de luxo.

0 objetivo desta pesquisa foi realizar um diagnóstico qualitativo dos parasitos encontrados em amostras fecais ambientais de jacarés (C. latirostris), criados comercialmente em sistema fechado, no estado do Rio de Janeiro, no período de 2008 a 2009.

\section{MATERIAL E MÉTODOS}

O criatório comercial localiza-se no estado do Rio de Janeiro e adota o sistema farming, ou seja, possui ciclo fechado de criação em tanques, sendo as fêmeas que originaram as criações capturadas de ambiente natural no Pantanal do Mato Grosso do Sul. Neste sistema os jacarés têm acesso livre à água e ao solo, possui assistência médico-veterinária, presença de rodolúvio e pedilúvio e são afastados dos centros urbanos. No mesmo existem diferentes tipos de tanques retangulares, onde se pode observar que alguns animais estão alocados em tanques fechados tipo estufas, para manterem a temperatura $\left(\right.$ de $30-37^{\circ} \mathrm{C}$ ) e umidade alta $(>80 \%)$, ou seja, condições controladas de ambiência.

Classificaram-se os animais deste criatório por fases de produção e consequentemente por faixa etária entre um e 24 meses em tanques, onde os filhotes possuem idades entre um mês a 12 meses, os animais adultos em fase de engorda possuem idades entre 12 meses e 24 meses e os pertencentes aos tanques de reprodução idades superiores a 24 meses.

A dieta desses animais consta de refugos de aves, pescado e suínos de abatedouros, que são partes não vendáveis para o consumo humano, ofertadas entre 15 e 30 dias, conforme a digestão dos mesmos, variando a periodicidade de acordo com a fase de produção em que se encontra esse animal. A água ingerida pelos animais é a própria do tanque, que só é trocada duas vezes ao ano. Quanto à circulação de outros animais no ambiente, encontramos em alguns tanques peixes e tartarugas. Notabiliza-se também a presença do homem, seja para o tratamento ou alimentação desses animais.

Um total de 300 amostras (de 150 filhotes, 80 engorda e 70 reprodução) oriundas de $C$. latirostris foram coletadas ambientalmente, medida preventiva para minimizar o estresse dos animais e manter o nível de segurança do tratador/pesquisador, de forma aleatória, durante os meses de julho, agosto, setembro e outubro no ano de 2008, diretamente do chão dos locais onde os animais se aqueciam ao sol, seguindo o protocolo determinado por Hoffmann (1987), ou seja, sempre evitando a coleta de materiais com sujidades, retirando apenas as partes superiores e internas das amostras. As mesmas foram acondicionadas em potes coletores de fezes devidamente etiquetados, mantidos à temperatura de $4^{\circ} \mathrm{C}$ com auxílio de caixa térmica e gelo para o transporte, sendo levadas posteriormente para o Setor de Parasitologia, Laboratório de Sanidade Animal, Hospital Veterinário da Universidade Estadual do Norte Fluminense Darcy Ribeiro (UENF).

Cada amostra foi submetida aos métodos de análises qualitativas, flutuação (método de Willis-Mollay) e sedimentação simples (método de Lutz), de acordo com Hoffmann (1987), que são técnicas interligadas, ou seja, partem de uma mesma metodologia, ovos leves flutuam, ovos pesados sedimentam, em solução supersaturada de sal ou açúcar, sendo nesta pesquisa utilizado o açúcar. Após seu processamento, as mesmas foram conduzidas ao microscópio óptico (Bioval@ ou Quimis $®$ ), onde através de aumentos de 40x, 100x e 400x, os ovos/oocistos de parasitos presentes foram observados, analisados e qualitativamente diagnosticados (Hoffmann 1987).

Os resultados relativos aos diagnósticos foram analisados estatisticamente utilizando o teste do qui-quadrado $\left(\chi^{2}\right)$ (Vieira 1998).

Este estudo foi realizado com aprovação da Comissão de Ética de Uso de Animais da Universidade Estadual do Norte Fluminense Darcy Ribeiro, protocolo 058/2009-CEUA/UENF, tendo sido respeitados todos os princípios éticos na experimentação animal adotados pela Sociedade Brasileira de Ciência de Animais de Laboratório/Colégio Brasileiro de Experimentação Animal. 


\section{RESULTADOS}

Os resultados encontrados apresentam de um total de 300 amostras fecais, 145 (48,00\%) parasitadas, sendo que coccídeos mostram-se mais freqüentes entre estas amostras, enquanto o parasitismo por helmintos e demais protozoários ostenta menor frequência (Quadro 1).

A análise estatística realizada demonstrou após os resultados dos exames coproparasitológicos (flutuação e sedimentação simples), que a presença dos endoparasitos não estava relacionada com as fases de produção $(P>0,05)$, não revelando significância $\left(\mathrm{c}^{2}=1,10\right)$ (Quadro 2).

Quadro 1. Total de amostras ambientais coletadas e parasitadas nas diferentes fases de produção e ocorrência de gêneros observados através da metodologia de flutuação-sedimentação, de julho a outubro de 2008 , em criatório comercial (sistema fechado) de Caiman latirostris

\begin{tabular}{|c|c|c|c|c|}
\hline \multirow{2}{*}{$\begin{array}{l}\text { Fases de } \\
\text { produção }\end{array}$} & \multicolumn{2}{|c|}{ Número de amostras* } & \multirow{2}{*}{$\begin{array}{c}\text { Gêneros } \\
\text { observados }\end{array}$} & \multirow{2}{*}{$\begin{array}{c}\text { Ovos/oocistos } \\
\text { encontrados }\end{array}$} \\
\hline & Totais & Parasitadas & & \\
\hline \multirow[t]{3}{*}{ Filhotes } & 150 & 75 & Eimeria sp. $^{+++}$ & 50 \\
\hline & & & Isospora sp. ${ }^{+++}$ & 70 \\
\hline & & & Balantidium sp. $^{+}$ & 1 \\
\hline \multirow[t]{3}{*}{ Engorda } & 80 & 40 & Eimeria sp. $^{+++}$ & 14 \\
\hline & & & Isospora sp..+ ${ }^{++}$ & 35 \\
\hline & & & Balantidium sp. $^{+}$ & 1 \\
\hline \multirow[t]{4}{*}{ Reprodução } & 70 & 30 & Eimeria sp..$^{+++}$ & 10 \\
\hline & & & Isospora sp. ${ }^{++}$ & 18 \\
\hline & & & Acanthostomum sp. & 1 \\
\hline & & & Dujardinascaris sp..$^{+}$ & 1 \\
\hline Total (\%) & $\begin{array}{c}300 \\
(100,00 \%)\end{array}$ & $\begin{array}{c}145 \\
(48,00 \%)\end{array}$ & & \\
\hline
\end{tabular}

+ Visualização de menos de cinco ovos/oocistos por campo, ${ }^{++}$Visualização de cinco a 10 ovos/oocistos por campo, ${ }^{+++}$Visualização de mais de 10 ovos/oocistos por campo.

Quadro 2. Resultado do teste qui-quadrado em amostras fecais ambientais de jacaré-do-papo-amarelo (Caiman latirostris) em sistema fechado de criação comercial, nas suas diferentes fases de produção, após exames coproparasitológicos de flutuação-sedimentação

\begin{tabular}{lccc}
\hline \multirow{2}{*}{ Fases de produção } & \multicolumn{2}{c}{ Exame coproparasitológico* } & \multirow{2}{*}{ Total } \\
\cline { 2 - 3 } & Positivo & Negativo & \\
\hline Filhote & 75 & 75 & 150 \\
Engorda & 40 & 40 & 80 \\
Reprodução & 30 & 40 & 70 \\
Total & 145 & 155 & 300
\end{tabular}

$\chi^{2}=1,10(\mathrm{P}>0,05)$.

Quadro 3. Resultado do teste qui-quadrado em amostras fecais ambientais de jacaré-do-papo-amarelo (Caiman latirostris) em sistema fechado de criação comercial, nas suas diferentes fases de produção, após exames coproparasitológicos de flutuação positivos para Eimeria sp.

\begin{tabular}{lccc}
\hline \multirow{2}{*}{ Fases de produção } & \multicolumn{2}{c}{ Exame coproparasitológico* } & Total \\
\cline { 2 - 3 } & Positivo & Negativo & \\
\hline Filhote & 50 & 100 & 150 \\
Engorda & 14 & 66 & 80 \\
Reprodução & 10 & 60 & 70 \\
Total & 74 & 226 & 300
\end{tabular}

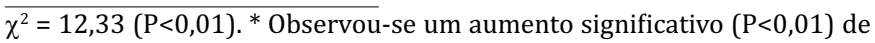
exames positivos para Eimeria sp. nos filhotes, enquanto que para os animais em reprodução observou-se uma redução significativa $(\mathrm{P}<0,05)$.
Quadro 4. Resultado do teste qui-quadrado em amostras fecais ambientais de jacaré-do-papo-amarelo (Caiman latirostris) em sistema fechado de criação comercial, nas suas diferentes fases de produção, após exames coproparasitológicos de flutuação positivos para Isospora sp.

\begin{tabular}{lccc}
\hline \multirow{2}{*}{ Fases de produção } & \multicolumn{2}{c}{ Exame coproparasitológico* } & Total \\
\cline { 2 - 3 } & Positivo & Negativo & \\
\hline Filhote & 70 & 80 & 150 \\
Engorda & 35 & 45 & 80 \\
Reprodução & 18 & 52 & 70 \\
Total & 123 & 177 & 300
\end{tabular}

$\chi^{2}=9,00(\mathrm{P}<0,05) .{ }^{*}$ Observou-se um aumento significativo $(\mathrm{P}<0,05)$ de exames positivos para Isospora sp. nos filhotes, enquanto que para os animais em reprodução observou-se uma redução significativa $(\mathrm{P}<0,01)$.

Quanto ao coccídio Eimeria sp., que apresentou grande quantidade, evidenciou-se após os resultados dos exames coproparasitológicos, que a presença do mesmo estava relacionada com as fases de produção $(\mathrm{P}<0,01)$, revelando significância $\left(\chi^{2}=12,33\right)$ (Quadro 3).

Para Isospora sp., que também apresentou relativa contagem, ficou comprovado após os resultados dos exames coproparasitológicos, que a sua presença estava relacionada igualmente com as fases de produção $(\mathrm{P}<0,05)$, revelando significância $\left(\chi^{2}=9,00\right)$ (Quadro 4).

No que se refere aos endoparasitos Balantidium sp., Acanthostomum sp. e Dujardinascaris sp., a análise estatística não foi realizada devido à baixa contagem nas amostras fecais ambientais diagnosticadas.

\section{DISCUSSÃO}

Por se tratarem de animais exclusivamente carnívoros, os jacarés (Caiman sp.) estão sujeitos a uma grande variedade de parasitismo ao capturarem e ingerirem suas presas praticamente inteiras, e com isso ocorre a ingestão de intestinos e outras partes do organismo que poderiam albergar uma ampla variedade de parasitos (Lavorenti \& Pinheiro 2001).

Considerando-se que é ofertada dieta à base de carne de frangos provenientes de mortes em aviários e/ou refugos de abatedouros, carne de suínos de mortalidade em maternidades e creches destes animais ou partes não aproveitadas de peixes e bovinos originárias de peixarias e abatedouros (Lavorenti \& Pinheiro 2001, Pinheiro \& Lavorenti 2001, Muller et al. 2005), torna-se mais provável ainda o risco de parasitismo gastrintestinal.

Muitas espécies de moscas podem veicular ovos e larvas de helmintos, tornando-se dípteros de considerável potencial contaminante de recintos de diversas espécies de animais, podendo algumas espécies hematófagas atacarem os crocodilianos (Ferreira et al. 2002, Oliveira et al. 2002).

Segundo os resultados obtidos neste trabalho, temperatura e umidade controlada, sempre em graus elevados, como acontece em estufas, podem favorecendo não somente o crescimento dos animais, mais também a proliferação e disseminação de oocistos de Eimeria sp. e Isospora sp. em seus tanques. 0 sistema fechado (estufas) impede a ação solar direta sobre os tanques, bem como promove um número maior de animais por tanque (superpopulação), podendo viabilizar desta forma a transmissão direta, de parasitos entre os répteis, uma vez que tais condições agem 
positivamente para o desenvolvimento de coccidioses (Urquhart et al. 1996).

Além disso, um motivo que também pode propiciar um maior grau de oocistos são as águas dos tanques, que costumam ser completadas quando necessário, e as trocas são realizadas em intervalos longos, em média a cada seis meses. 0 fator temperatura elevada nas estufas $\mathrm{x}$ fezes depositadas na água, torna-se mais propício ao desenvolvimento de parasitos. Se as estufas possuíssem cobertura de vidro, o que não ocorre, pois são de plástico, possivelmente ocorreria uma ação dos raios solares viabilizando a minimização do número de parasitos.

Alguns dos tanques possuem peixes coabitando com os filhotes de jacarés, tendo por finalidade se alimentarem das fezes dos jacarés, semelhante ao que ocorre na natureza. Porém, nas condições em que se encontram, ou seja, em tanques restritos, os peixes por serem animais que possuem potencial de albergar uma infinidade de nematóides, podem se comportar como reservatórios e disseminadores de parasitoses (Urquhart et al. 1996, Foreyt 2005, Menezes et al. 2006).

No presente trabalho os coccídeos se apresentaram estatisticamente em maior grau de parasitismo, onde predominaram os gêneros Eimeria e Isospora nos tanques de filhotes e de engorda. Observou-se que o controle da ambiência associado à superpopulação em tanques principalmente de filhotes acarreta em um maior parasitismo, e esse fato corrobora com diversos autores (Gennari et al. 1999, Sequeira \& Amarante 2002, Freitas et al. 2003, Foreyt 2005, Silva et al. 2008), quando pesquisaram a presença de coccídeos em fezes de animais criados em sistema intensivos, ou seja, confinados.

Outro gênero de parasito diagnosticado foi o Balantidium sp., que se trata de um protozoário intestinal preferencialmente do homem e de suínos, também já observado em peixes, aves e suínos silvestres (Mundin et al. 2004). A presença deste gênero de parasito sugere que estes animais possam ter se infectado ao ingerir carne contaminada. Outro fator que também vai de encontro com a positividade deste resultado para Balantidium sp. é que em um dos tanques onde ficam os filhotes, existe a presença de tartaruga, uma vez que este gênero de parasito é encontrado em tartarugas e cágados, sugere a possibilidade de contaminação cruzada, segundo Foreyt (2005).

Nesta pesquisa também foi diagnosticado o gênero Acanthostomum sp. Este gênero de parasito é um trematóide já identificado em jacarés e crocodilos, sendo parasitas do intestino destes répteis (Catto 1991, Catto \& Amato 1993, Moravec 2001, Fernandes et al. 2002, Tkach \& Snyder 2003, Foreyt, 2005). Exemplares do gênero Acanthostomum sp. foram observados parasitando répteis e peixes de água doce e da costa marinha do Brasil e de outros países (Karyakarte 1967, Fernandes et al. 2002, Corrêa \& Brasil-Sato 2008).

0 resultado para este gênero de parasito neste trabalho se deu apenas em um dos tanques de reprodução, e aparentemente muito baixo, sugerindo que por algum momento possam ter sido ofertados peixes na dieta dos animais destes tanques, uma vez que como dito anteriormente, peixes também fazem parte da dieta destes répteis, tanto na natureza quanto em cativeiro (Lavorenti \& Pinheiro 2001).

Dujardinascaris sp. já foi identificado parasitando diferentes espécies de crocodilianos, como por exemplo, crocodilos africanos e asiáticos. Ainda não estão muito bem elucidados todos os aspectos biológicos deste gênero de parasito, porém, estudos demonstram ser este, um parasito de ciclo de vida indireto, ou seja, necessita de hospedeiros intermediários que desenvolvam a forma infectante (L3) para seus hospedeiros, tais como peixes e anfíbios que nestas condições, passam a servir de fonte de infecção aos jacarés, que se alimentam destes animais praticamente inteiros e crus (Catto 1991, Moravec 2001). Tais condições sugerem ser a hipótese desta via de infecção totalmente viável para jacarés criados em cativeiro e que recebem esporadicamente peixes em sua dieta.

\section{CONCLUSÕES}

De acordo com nossa pesquisa, verificamos a ocorrência dos gêneros de parasitos Acanthostomum, Balantidium, Dujardinascaris, Eimeria e Isospora, em jacaré-do-papo-amarelo (C. latirostris) criados comercialmente em sistema fechado.

O foco deste trabalho não está voltado para a mensuração do bem estar animal e sim para a pesquisa parasitológica, mas isso não nos impede de supormos que este parasitismo seja proveniente de pressão de estresse (superpopulação) e alimentação (refugos de carcaças). Hipotetizamos também, que a transmissão parasitária ocorra de duas formas, através das matrizes e reprodutores oriundos de regiões nascedouras (como do Pantanal/MS e de Jacarepaguá/RJ), onde poderiam ter sido acometidos por uma gama de parasitos e ao serem transportados para outros lugares carrearam para novo ambiente, ou intracriatória.

No que diz respeito à transmissão parasitária intracriatória, diversos fatores extrínsecos e intrínsecos podem estar aí relacionados, entre eles, oferta alimentar (carcaças animais, refugos de vísceras e água), predativismo (relação caça/predador) e manejo inadequado (criação alimentar estratégica, presença de animais, vetores e homem, troca de água, controle da ambiência e superpopulação).

A realização de exames coproparasitológicos rotineiros em criatórios deveria ser uma prática comum, pois além de permitir realizar o diagnóstico real de seus animais, permite investigar as causas e adequar seus sistemas de manejo para obtenção de melhores resultados, isto é, higidez animal.

Neste estudo não podemos afirmar qual é a relação de parasitismo preponderante, se este animal comporta-se como hospedeiro definitivo, intermediário ou paratênico, havendo necessidade do estabelecimento de ciclos biológicos.

A parasitose clínica nesses animais não é observada devido a diversos fatores, mas principalmente por ser essa espécie a mais antiga do mundo. 0 seu poder de adaptação, resposta imunológica e metabólica, é ainda um mistério para o homem. Faz-se necessário, nesse sentido, que avancem pesquisas a respeito desses animais, inclusive para utilização dos meios de autocura no tratamento de doenças que acometem a espécie humana. 


\section{REFERÊNCIAS}

Catto J.B. 1991. Taxionomia e ecologia dos helmintos parasitos de Caiman crocodilus yacare. Tese de Doutorado em Medicina Veterinária, Seropédica RJ. 125p.

Catto J.B. \& Amato F.R. 1993. Digenetic trematodes (Cryptogonimidae, Acanthostominae) parasites of the caiman, Caiman crocodilus yacare (Reptilia, Crocodylia) from the Pantanal Mato-Grossense, Brazil, with the description of a new species. Mem. Inst. Oswaldo Cruz 88:435-440.

Corrêa R.F.S.S. \& Brasil-Sato M.C. 2008. Digenea in the Surubim Pseudoplatystoma corruscans (Spix and Agassiz, 1829) (Siluriformes: Pimelodidae) of the upper São Francisco River, states of Minas Gerais, Brazil. Braz. Arch. Biol. Technol. 51:1181-1185.

Dias R.J.P., Almeida S.J.B., Prieto D.B. \& Lima S.S. 2004. Aspectos ecológicos dos nematóides parasitos de Crotalus durissus terrificus Laurenti, 1768 (Ophidia, Viperidae), em Juiz de Fora, Minas Gerais, Brasil. Revta Bras. Zool. 6:231-235.

Fernandes B.M.M., Pinto R.M. \& Cohen S.C. 2002. Report on two species of Digenea from marine fishes in Brazil. Braz. J. Biol. 62:459-462.

Ferreira R.L.M., Henriques A.L. \& Rafael J.A. 2002. Activity of tabanids (Insecta: Diptera: Tabanidae) attacking the reptiles Caiman crocodilus (Linn.) (Alligatoridae) and Eunectes murinus (Linn.), in the Central Amazon, Brazil. Mem. Inst. Oswaldo Cruz 97:133-136.

Foreyt W.J. 2005. Parasitologia Veterinária. Roca, Rio de Janeiro. 226p.

Freitas M.F.L., De Oliveira J.B., De Brito M.C. \& Djalma A.F. 2003. Occurrence of coccidiosis in canaries (Serinus canarius) being kept in private captivity in the state of Pernambuco, Brazil. Parasitol. Latinoam. 58:86-88.

Gennari S.M., Kasai N., Pena H.F.J. \& Cortez A. 1999. Ocorrência de protozoários e helmintos em amostras de fezes de cães e gatos da cidade de São Paulo. Braz. J. Vet. Res. Anim. Sci. 36:87-91.

Hoffmann R.P. 1987. Diagnóstico de Parasitismo Veterinário. Sulina, Rio de Janeiro. 156p.

Huchzermeyer F.W. 2003. Crocodiles: Biology, husbandry and diseases. CABI Publishing, Cambridge. 337p.

Jaques A.M.C.C. 2011. Avaliação macroscópica e microscópica de vísceras de Jacaré-açu (Melanosuchus niger Spix, 1825) abatidos na reserva de desenvolvimento sustentável Mamirauá e destinados ao consumo humano. Tese de Doutorado em Higiene Veterinária e Processamento Tecnológico de Produtos de Origem Animal, Niterói RJ. 68p.

Karyakarte P.P. 1967. Acanthostomum (Atrophecaecum) alli sp. n. (Trematoda: Acanthostomidae) from the snake, Elaphe helena, in India. J. Parasitol. 53:587-588.

Lavorenti A. \& Pinheiro M.S. 2001. Growth of broad-nosed caiman, Caiman latirostris (Daudin, 1802) hatchlings, fed with diets of animal origin. Braz. J. Biol. 61:421-429.

Menezes R.C., Tortelly R., Neto R.T., Noronha D. \& Pinto R.M. 2006. Camallanus cotti Fujita, 1927 (Nematoda, Camallanoidea) in ornamental aquarium fishes: pathology and morphology. Mem. Inst. Oswaldo Cruz 101:683-687.

Moravec F. 2001. Some helminth parasites from Morelet's crocodile, Crocodylus moreletii, from Yucatan, Mexico. Folia Parasitol. 48:47-62.

Mourão G.M. \& Campos Z.M.S. 2004. Biologia reprodutiva de jacaré-de-papo-amarelo no rio Paraná, Brasil. Embrapa, Paraná. 25p.

Muller G.C.H., Greinert J.A. \& Silva Filho H.H. 2005. Freqüência de parasitas intestinais em felinos mantidos em zoológicos. Arq. Bras. Med. Vet. Zootec. 57:559-561.

Mundin M.J.S., Mundin A.V., Santos A.L.Q., Cabral D.D., Faria E.S.M. \& Moraes F.M. 2004. Helmintos e protozoários em fezes de javalis (Sus scrofa scrofa) criados em cativeiro. Arq. Bras. Med. Vet. Zootec. 56:792-795.

Oliveira V.C., Mello R.P. \& D'Almeida J.M. 2002. Muscoid dipterans as helminth eggs mechanical vectors at the zoological garden, Brazil. Revta Saúde Pública 36:614-620.

Panizzutti M.H.M., Santos L.C., Vicente J.J., Pereira L.C.M. \& Pinto R.M. 2003. Ophidascaris durissus spp. (Nematoda, Ascarididae) parasitizing Crotalus durissus Linnaeus (Ophidia, Viperidae) in Brazil. Revta Bras. Zool. 20:9-11.

Pinheiro M.S. \& Lavorenti A. 2001. Growth of broad-nosed caiman, Caiman latirostris (Daudin, 1802) hatchlings, fed with diets of animal origin. Braz. J. Biol. 61:421-429.

Sarkis-Gonçalves F., Miranda-Vilela M.P., Bassetti L.A.B. \& Verdade L.M. 2001. Manejo de jacarés-de-papo-amarelo (Caiman latirostris) em cativeiro, p.565-578. In: Mattos W.R.S. (Ed.), A Produção Animal na Visão dos Brasileiros. Sociedade Brasileira de Zootecnia, Brasília.

Sequeira T.C.G.O. \& Amarante F.T. 2002. Parasitologia Animal. EPUB, São Paulo. 149p.

Silva A.S., Coradini G.P., Gressler L.T., Soares J.F., Lara V.M., Carregaro A.B. \& Monteiro S.G. 2008. Ocorrência de protozoários gastrintestinais em primatas mantidos em cativeiro na região sul do Brasil. Ciência Rural 38:2658-2661.

Tkach V.V. \& Snyder S.D. 2003. Acanthostomum macroclemidis n. sp. (Digenea: Cryptogonimidae: Acanthostominae) from the alligator snapping turtle, Macroclemys temmincki. J. Parasitol. 89:159-167.

Urquhart A.M., Armour J., Duncan J.L., Dunn A.M. \& Jennings F.W. 1996. Parasitologia Veterinária. Guanabara Koogan, Rio de Janeiro. 135p.

Vieira S. 1998. Introdução à Bioestatística. Editora Campos, Rio de Janeiro. $196 \mathrm{p}$. 